\title{
Mitogen-activated protein kinases (MAPKs) are modulated during Francisella tularensis infection, but inhibition of extracellular-signal-regulated kinases (ERKs) is of limited therapeutic benefit
}

\author{
R. J. Saint ${ }^{1}$ - R. V. D'Elia ${ }^{1}$ - C. Bryant ${ }^{2}$ - G. C. Clark $^{1}$ - H. S. Atkins ${ }^{1,3}$
}

Received: 13 May 2016/Accepted: 9 August 2016/Published online: 6 October 2016

(C) Crown Copyright, Dstl 2016

\begin{abstract}
Francisella tularensis is a Gram-negative intracellular bacterium that causes the disease tularemia. The disease can be fatal if left untreated and there is currently no licenced vaccine available; the identification of new therapeutic targets is therefore required. Toll-like receptors represent an interesting target for therapeutic modulation due to their essential role in generating immune responses. In this study, we analysed the in vitro expression of the key mitogen-activated protein kinases (MAPKs) p38, JNK and ERK in murine alveolar macrophages during infection with $F$. tularensis. The phosphorylation profile of ERK highlighted its potential as a target for therapeutic modulation and subsequently the effect of ERK manipulation was measured in a lethal intranasal $F$. tularensis in vivo model of infection. The selective ERK1/2 inhibitor PD0325901 was administered orally to mice either pre- or post-challenge with $F$. tularensis strain LVS. Both treatment regimens selectively reduced ERK expression, but only the pre-exposure treatment produced decreased bacterial burden in the spleen and liver, which correlated with a significant reduction in the pro-inflammatory cytokines IFN- $\gamma$, MCP-1, IL-6, and TNF- $\alpha$. However, no overall improvements in survival were observed for treated animals in this study. ERK may represent a useful therapeutic target where selective dampening of the immune response (to control the damaging pathology seen during infection) is combined with antibiotic
\end{abstract}

R. V. D'Elia

rvdelia@dstl.gov.uk

1 CBR Division, Defence Science and Technology Laboratory, Porton Down, Salisbury SP4 0JQ, UK

2 Department of Veterinary Medicine, University of Cambridge, Madingley Road, Cambridge CB3 0ES, UK

3 University of Exeter, Exeter, UK treatment required to eradicate bacterial infection. This combination treatment strategy has been shown to be effective in other models of tularemia.

\section{Introduction}

Toll-like receptors (TLRs) are sentinels of the innate immune system, functioning to detect invading microorganisms by binding to pathogen-associated molecular patterns (PAMPs) such as lipids, proteins and nucleic acids [1-3]. The binding event triggers an intracellular signalling cascade through dimerisation of the receptor and the subsequent recruitment of signalling proteins, leading to the production of immune mediators such as chemokines and cytokines. Eleven TLRs have been identified in humans located on multiple cell types including neutrophils, macrophages, and epithelial cells $[4,5]$. TLR activity is vital for pathogen clearance, but dysregulation can occur which can have severe consequences for the host. Indeed, it is now well documented that an overactive immune response can be more damaging to the host than the progression of infection by the pathogen [6]. This fine balance is illustrated when a host is infected via the inhalational route with the Gram-negative facultative intracellular bacterium Francisella tularensis, where there is an initial suppression of pro-inflammatory cytokines followed by a "cytokine storm" prior to death [7].

The primary detection of $F$. tularensis was originally thought to be mediated by TLR4 $[8,9]$. However, more recent studies demonstrate that it is likely that TLR2, and not TLR4, confers the ability to detect $F$. tularensis [10-12]. TLR4 was ruled out as a major receptor in the detection of $F$. tularensis as TLR4-defective mice were no more susceptible to an inhalational infection with $F$. tularensis than wild-type mice and the survival rates were similar in both mouse strains [13]. In 
contrast, several studies have reported a requirement for TLR2 in the detection of $F$. tularensis following in vivo infection by multiple challenge routes or in different types of cultured cell [10, 14-19]. More recently, the importance of TLR2-mediated priming of the inflammasome to intracellular survival has been highlighted [20]. Furthermore, a TLR2 deficiency enhanced murine susceptibility to pulmonary infection by the F. tularensis live vaccine strain (LVS) and resulted in decreased production of pro-inflammatory cytokines including TNF- $\alpha$ and IL-6 [10].

A number of studies have previously demonstrated the potential therapeutic benefits of specifically targeting TLRs for the treatment of infectious diseases including those caused by Francisella $s p$. For example, bacterial DNA containing unmethylated $\mathrm{CpG}$ motifs have been used to stimulate TLR9 and induce protection against subsequent challenge with the intracellular pathogens $F$. tularensis strain LVS and Listeria monocytogenes [21]. Furthermore, stimulation of TLR3 using the synthetic dsRNA analogue poly(I:C) has been evaluated for the treatment of $F$. tularensis strain LVS or strain SchuS4 infection in Balb/c mice [22]. Treatment as a monotherapy $1 \mathrm{~h}$ prior to or $1 \mathrm{~h}$ after intranasal infection led to significant improvements in the survival of mice, correlating with an increased influx of neutrophils to the lungs and early upregulation of cytokines IL-6, MCP-1, and TNF- $\alpha$ [22]. However, it is noteworthy that a combination therapy approach with the antibiotic levofloxacin was required for full recovery.

In comparison to TLR receptors, less research has focused on understanding the activation and phosphorylation states of the downstream immune signalling cascade proteins during F. tularensis infection and/or the potential for selectively targeting these pathways with therapeutics. Previously, it has been demonstrated that the up-regulation of $\mathrm{p} 38$, IkB and cJun occurs following infection of murine macrophages with F. tularensis strain LVS [15]. In addition, it has been demonstrated that the uptake of Francisella novicida by macrophages is dependent upon ERK activation, and modulation of the MAPK pathways is important for intracellular survival of $F$. tularensis LVS $[23,24]$. Furthermore, within an in-vivo setting it was found that the inhibition of GSK3B (a serine/ threonine protein kinase) with lithium chloride significantly reduces the production of pro-inflammatory cytokines such as IFN- $\gamma$, IL-12p40, and TNF- $\alpha$ in F. tularensis LVS-infected mice, and increases survival from $60 \%$ in untreated mice to $90 \%$ following treatment [25]. It is hypothesised that targeting specific proteins within the TLR signalling cascades may prevent the wide-ranging effects of direct TLR stimulation. In this study we sought to determine the in vitro expression profile of key mitogen-activated protein kinases (MAPKs) p38, JNK, and ERK following F. tularensis infection. Additionally, we determined the effect of inhibiting ERK expression in vitro and in vivo during a $F$. tularensis infection.

\section{Materials and methods}

\section{Preparation of bacteria}

Francisella tularensis live vaccine strain (LVS) was grown overnight on blood cysteine glucose agar (BCGA) plates incubated at $37^{\circ} \mathrm{C}$, and used to inoculate $10 \mathrm{ml} \mathrm{PBS}$ to an $\mathrm{OD}_{600}$ of $0.15\left(\sim 1 \times 10^{9} \mathrm{CFU} / \mathrm{ml}\right)$. The resulting bacterial suspension was diluted to the required inoculum. To confirm the CFU/ml of the inoculum, serial dilutions were cultured on BCGA plates for subsequent bacterial enumeration.

\section{Infection of MH-S cell line with $F$. tularensis LVS}

A continuous cell line of murine alveolar macrophages (MH-S cells, ECACC UK) were seeded into 6-well plates (Corning, US) at $\sim 4 \times 10^{5}$ cells $/ \mathrm{ml}$ and incubated overnight at $37{ }^{\circ} \mathrm{C}$ to allow the cells to adhere. Supernatants were then removed and replaced with $1.5 \mathrm{ml}$ of $F$. tularensis LVS inoculum to generate an MOI of 100 . Plates were incubated at $37^{\circ} \mathrm{C}$ for $2 \mathrm{~h}$ to allow for internalisation of the bacteria, and then the supernatants were removed and replaced with $1.5 \mathrm{ml}$ fresh media. The plates were incubated at $37^{\circ} \mathrm{C}$ and, at a range of timepoints, supernatants were removed and stored at $-20^{\circ} \mathrm{C}$ for cytokine analysis. The cells were washed once with chilled PBS and then lysed by adding $350 \mu$ l PhosphoSafe ${ }^{\mathrm{TM}}$ extraction reagent (Merck, US) before incubating on ice for $5 \mathrm{~min}$. Full cell recovery was ensured by scraping wells with a rubber policeman (Corning, US): $100 \mu$ of the lysate was used for bacterial enumeration, and a further $250 \mu \mathrm{l}$ was centrifuged at $13,000 \mathrm{rpm}$ for $5 \mathrm{~min}$ to remove cell debris before being stored at $-20^{\circ} \mathrm{C}$ for analysis by western blotting.

\section{In-vivo infection and treatment with ERK inhibitor PD0325901}

A total of 65 female 6- to 8-week-old Balb/c mice (Charles River Laboratories, UK) were separated into four groups: group I (20 mice), group II (20 mice), group III (15 mice) and group IV (10 mice). All mice, except those in group IV, were treated daily with either PBS or $0.05 \mu \mathrm{M}$ PD0325901 (InvivoGen, France) by oral-gavage in a volume of $100 \mu \mathrm{l}$. Groups I and II were treated from $24 \mathrm{~h}$ pre-infection, while group III was treated from $48 \mathrm{~h}$ post-infection. The mice were infected via the intranasal route with approximately $2.5 \times 10^{4} \mathrm{CFU}$ of $F$. tularensis strain LVS. Scoring of the mice occurred twice daily throughout the study. At $48 \mathrm{~h}$ and $96 \mathrm{~h}$ post-challenge, five mice from each of groups I, II, III, and IV were culled and the lungs, liver, and spleen were removed and placed into aliquots of $2 \mathrm{ml}$ PBS. Ten mice from each of groups I, II, III, and IV were monitored for changes in survival. Organs were homogenised though a $0.2 \mu \mathrm{m}$ cell sieve (BD Biosciences) and screened for the levels of cytokines, 
activation of signalling proteins and bacterial enumeration on agar. All animal studies were carried out in accordance with the UK Scientific Procedures Act (1986). Animal handling and infection procedures were carried out by trained staff at Dstl.

\section{Western blots}

Proteins were separated on a 4-20\% tris-glycine polyacrylamide gel and then transferred onto Invitrolon ${ }^{\mathrm{TM}}$ polyvinylidene fluoride (PVDF) membranes (Invitrogen) using a Novex ${ }^{\circledR}$ Semi-Dry Blotter (Invitrogen) according to manufacturer's instructions. The PVDF membranes were then incubated in blocking buffer $(5 \%[\mathrm{w} / \mathrm{v}]$ skimmed milk powder and $0.1 \%$ Tween ${ }^{\circledR} 20$ (Sigma-Aldrich) dissolved in PBS) for $2 \mathrm{~h}$ at room temperature. To detect ERK activation, the membranes were washed in wash buffer $\left(0.1 \%\right.$ Tween ${ }^{\circledR} 20$ in PBS) before being incubated overnight at $4{ }^{\circ} \mathrm{C}$ with the primary antibody [p38 - \#9218; P-p38 - \#9211; SAPK/JNK \#9252; P-SAPK/JNK - \#9251; ERK-1 - \#4372; ERK-2 \#9108; P-ERK-1/2 - \#9101 (NEB)] at a dilution of 1:1,000 in blocking buffer. The membranes were then rinsed in wash buffer and incubated for $1 \mathrm{~h}$ at room temperature with the secondary antibody [goat anti-rabbit IgG conjugated to horseradish peroxidise (Bio-Rad)] at a dilution of 1:3,000 in blocking buffer. The membranes were rinsed in wash buffer and developed using enhanced chemiluminescence (ECL)Plus detection reagents (GE Healthcare) according to manufacturer's instructions. The membranes were placed in a film cassette and exposed to ECL hyperfilm (GE Healthcare) which was allowed to air-dry before analysis by densitometry.

\section{Cytometric bead array}

The quantities of the cytokines IFN- $\gamma$, MCP-1, IL-6, IL12 p70, IL-10, and TNF- $\alpha$ present within the lungs were measured using $\mathrm{BD}^{\mathrm{TM}}$ cytometric bead array (CBA) technology (BD Biosciences) on a $\mathrm{BD}^{\mathrm{TM}}$ FACScan flow cytometer (BD Biosciences) according to manufacturer's instructions. A standard curve was generated and the concentration of each cytokine was interpolated using GraphPad Prism ${ }^{\circledR} 5.01$ software (GraphPad). The levels of IL-12p70 and IL-10 were too low to be detected, and so only the results of IFN- $\gamma$, MCP-1, IL-6, and TNF- $\alpha$ are shown.

\section{Statistical analysis}

All transformations of data and statistical tests were performed using GraphPad Prism ${ }^{\circledR} 5.01$ software. Data from cytometric bead arrays were analysed using PRISM, by fitting a quadratic regression to the standard curves and reading the samples as unknowns.

\section{Results}

ERK, p38, and JNK represent key signalling molecules in the MAPK cascade [26]. Activation of these proteins, by phosphorylation, has diverse biological effects including cell proliferation, apoptosis, and inflammation [27]. In this study, temporal changes in the expression of total ERK, p38, and JNK and the phosphorylated forms of the proteins produced by in vitro cultured alveolar macrophage (MH-S) cell line in response to infection with $F$. tularensis LVS was measured via western blot.

The in vitro expression of p38 remained constant during the infection (Fig. 1a). However, there was a significant change in the p38 activation profile. First, a rapid increase in activation occurred immediately after the uptake of LVS; this was a transient response, and the quantities of activated protein present returned to naïve levels by $0.5 \mathrm{~h}$ (Fig. 1b). After the rapid and transient activation of p38 there was no reactivation measured throughout the rest of the infection assay. In comparison, limited in vitro activation of JNK proteins was observed during the experiment, with the exception of the 24-h time point, where there was a decrease in the presence of activated JNK-2/3 compared to that found at both $0.75 \mathrm{~h}$ and $6 \mathrm{~h}$ (Fig. 2d). The expression of total JNK-2/3 remained relatively constant (Fig. 2b), although there was a slight decrease in the expression of total JNK-1 from 6 to $24 \mathrm{~h}$ postuptake (Fig. 2a).

The in vitro expression of total ERK-1 and ERK-2 did not alter significantly at any time point during the infection (Fig. 3a, b). The activation profiles of the two proteins did, however, display some interesting features (Fig. 3c, d). There appeared to be two phases of protein activation, particularly for ERK-2 (Fig. 3d). In the first hour after uptake a transient activation was observed. However, from $1.5 \mathrm{~h}$ there was a sustained activation of both ERK proteins which was maintained through to $6 \mathrm{~h}$.

The interesting phosphorylation profile of ERK $1 / 2$ following $F$. tularensis LVS infection in vitro led us to consider modulation of the ERK proteins as an approach for treating infection. An inhibitor of ERK PD0325901 was identified and assessed in vivo for its potential as a novel therapy. PD0325901 is a small molecule inhibitor with specific activity against phospho-ERK1/2 and increased potency compared to its predecessors $[28,29]$. First, we demonstrated that PD0325901 was able to inhibit ERK expression during an in vitro $F$. tularensis macrophage infection assay and that it had a biological effect by significantly reducing the secretion of TNF- $\alpha$ (data not shown).

Subsequently, the potential for ERK inhibition (using PD0325901) was explored in vivo using a $F$. tularensis LVS intranasal model of infection. Balb/C mice infected with F. tularensis LVS showed a substantial increase in the level of ERK activation between $48 \mathrm{~h}$ and $96 \mathrm{~h}$, indicating the 
Fig. 1 The expression and the levels of activated p38 in MH-S cells throughout the course of a F. tularensis LVS infection. The expression of $\mathrm{p} 38$ (a) and the levels of activated p38 (b) were determined at several time points after the 2-h uptake period. Representative western blots are shown below the corresponding graphs. The experiment was performed in triplicate $(n=3)$ and each replicate was performed in duplicate. Error bars show the $95 \%$ confidence interval. Significant difference from naïve levels was determined by oneway ANOVA and a Dunnett's multiple comparison post-test $(*=p<0.05)$. Western blot image: $L-$ MagicMark $^{\mathrm{TM}}$ ladder; $N$ - naïve; +ve

- positive control

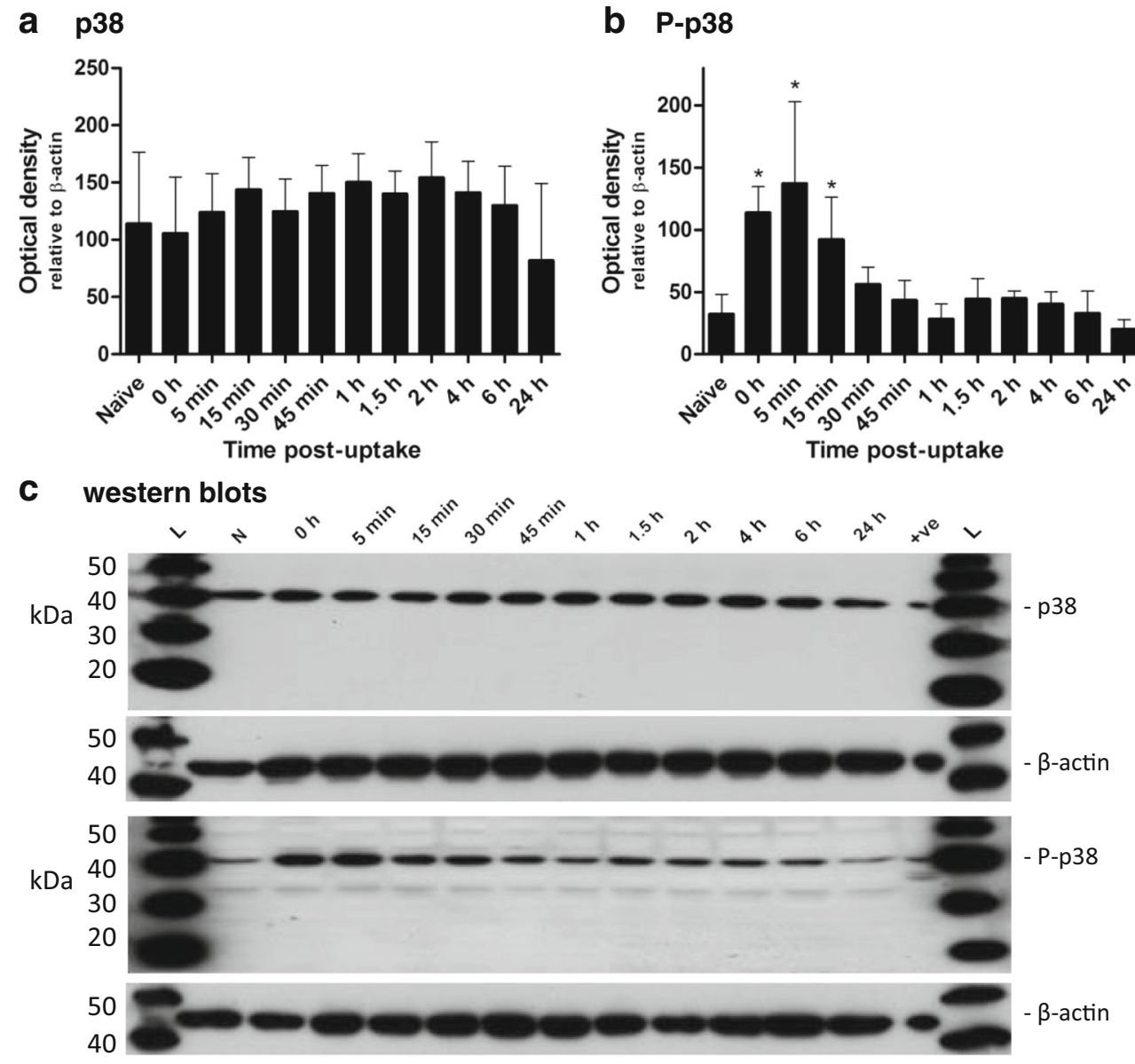

involvement of ERK phosphorylation during infection in vivo, similarly to in vitro. Daily treatment of infected mice with PD0325901 significantly reduced both ERK-1 and ERK2 activation in the lungs compared to PBS-treated mice at $96 \mathrm{~h}$ (Fig. 4a, b). This was found for both the PD0325901 treatment groups, irrespective of whether administration began at $24 \mathrm{~h}$ pre-infection or at $48 \mathrm{~h}$ post-infection. However, unexpectedly, at $48 \mathrm{~h}$ PD0325901-treated mice appeared to show an increase in ERK activation when compared to PBS-treated mice.

To examine the effect of ERK inhibition on bacterial loads in the organs of infected mice, bacterial enumeration was carried out on samples taken from the lungs, liver, and spleens at $96 \mathrm{~h}$ post-infection (Fig. 5a). There was no significant difference in the numbers of bacteria present in the lungs of either of the PD0325901-treated groups compared to PBS-treated controls. However, there was a significant reduction in bacterial burden in the liver and spleen of mice treated with PD0325901 prior to infection when compared to PBStreated control mice. These differences in the spleen and liver were not detected in mice treated with PD0325901 at $48 \mathrm{~h}$ post-infection.
The effect of ERK inhibition on the inflammatory response was examined by measuring the secretion of downstream inflammatory cytokines in the lungs, liver, and spleen of naïve, PBS-treated or PD0325901-treated mice at $96 \mathrm{~h}$ after infection (Fig. 5b-e). Measurable levels of IL-6 (Fig. 5b) MCP-1 (Fig. 5c), IFN- $\gamma$ (Fig. 5d) and TNF- $\alpha$ (Fig. 5e) were detected, but levels of IL-12p40 or IL-10 were below the limit of detection in all samples. At $96 \mathrm{~h}$, no reduction in secretion of any of the cytokines was found in the lungs, despite the observed inhibition of ERK activation in the lungs of mice (Fig. 4). Mice treated from $48 \mathrm{~h}$ post-infection with PD0325901 displayed a significant increase in IFN- $\gamma$ and MCP-1 in livers when compared to PBS-treated mice. The greatest effect of ERK inhibition was observed in spleen samples, where there was a significant decrease in the secreted levels of IFN- $\gamma$, MCP-1, IL-6, and TNF- $\alpha$ in mice treated with PD0325901 prior to infection (at day -1 ) compared to PBS-treated controls. This difference in the spleen was not seen when PD0325901 was administered at $48 \mathrm{~h}$ post-infection. Despite the PD0325901-associated inhibition of ERK activation within the lungs and the reduced bacterial burdens in the spleens and livers of infected mice, there was no significant difference 
Fig. 2 The expression and the levels of activated JNK-1 and JNK-2/3 in MH-S cells throughout the course of a F. tularensis LVS infection. The expression of JNK-1 (a) and JNK-2/3 (b) and the levels of activated JNK-1 (c) and JNK-2/3 (d) were determined at several time points after the 2-h uptake period. Representative western blots are shown below the corresponding graphs. The experiment was performed in triplicate $(n=3)$ and each replicate was performed in duplicate. Error bars show the $95 \%$ confidence interval. Significant difference from naïve levels was determined by oneway ANOVA and a Dunnett's multiple comparison post-test $(*=p<0.05)$. Western blot image: $L-$ MagicMark $^{\mathrm{TM}}$ ladder; $N$ - naïve; $+v e-$ positive control a

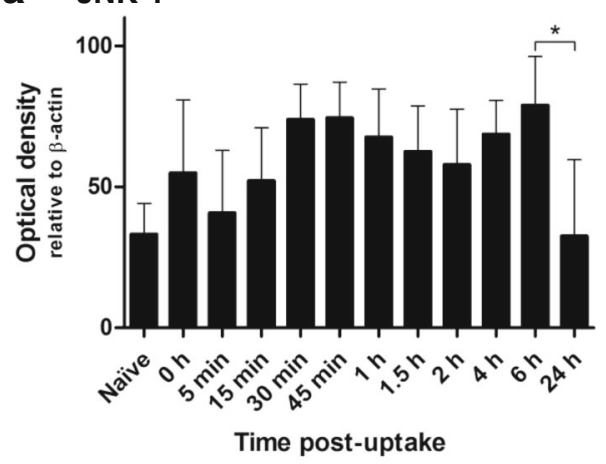

b JNK-2/3

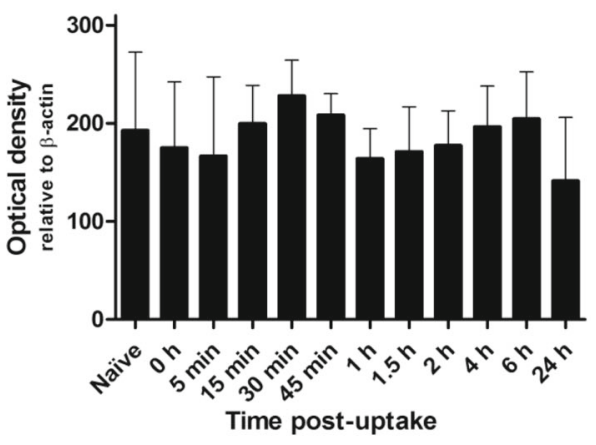

C western blots

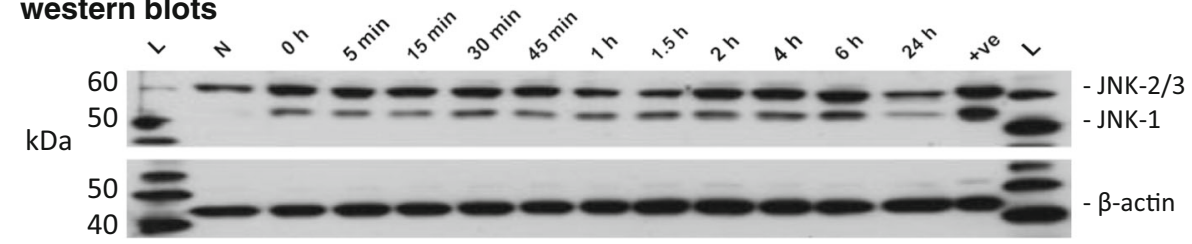

d P-JNK-1

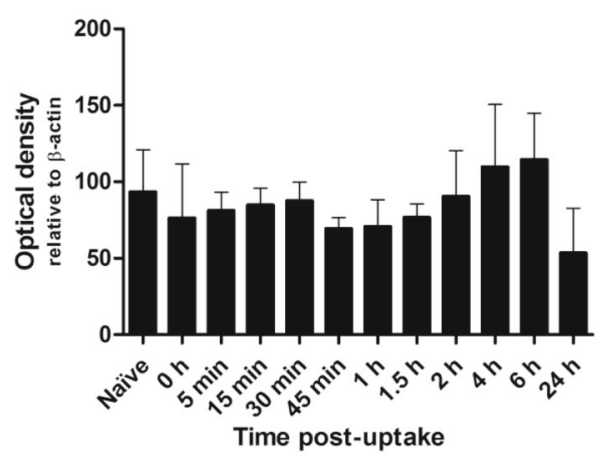

e P-JNK-2/3

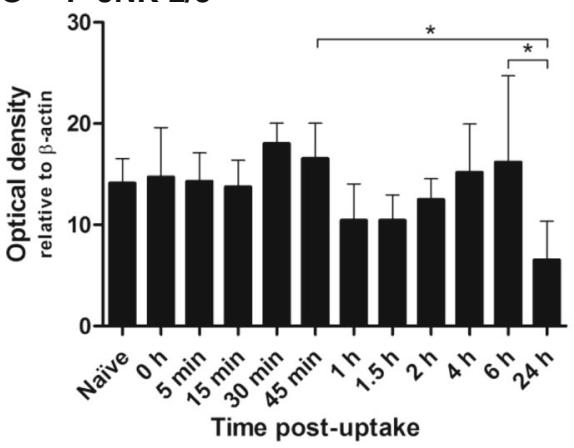

f

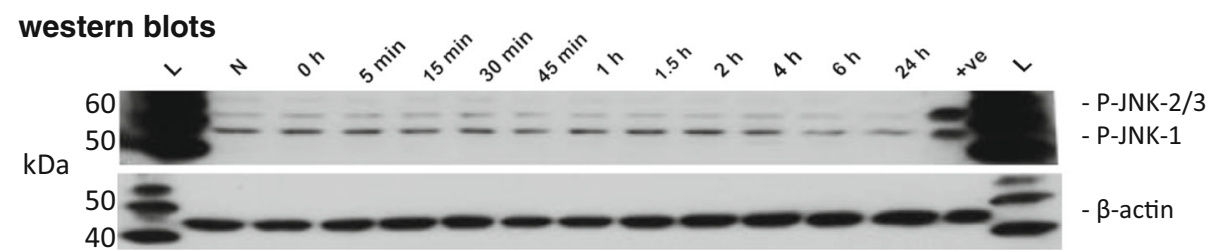

in survival of PD0325901-treated groups compared to the PBS-treated groups (Fig. 6).

\section{Discussion}

Antibiotics remain the most successful therapeutic regimen for F. tularensis infection, although efficacy is dependent on the correct administration of antibiotic in a timely manner [30]. Aiding antibiotic efficiency through immunemodulation is a strategy that is being explored for infectious diseases.

In these studies, we have demonstrated that total expression of the key MAPKs p38, JNK, and ERK were not significantly altered in an in vitro macrophage model of $F$. tularensis LVS infection. However, clear temporal changes in the phosphorylated states of the MAPKs were identified. Notably, the in vitro phosphorylation profile of ERK occurred in two distinct peaks of activation within infected macrophages. In addition, we identified that ERK was also significantly upregulated in vivo following intranasal infection of mice with F. tularensis LVS. By administering an inhibitor of ERK phosphorylation commencing both pre- and post-challenge, we aimed to evaluate this as a therapeutic strategy for treating infections caused by this organism. Following oral administration of the inhibitor to mice, a reduction of ERK phosphorylation was successfully achieved in vivo, with the greatest reductions observed when the compound was given prior to 
a

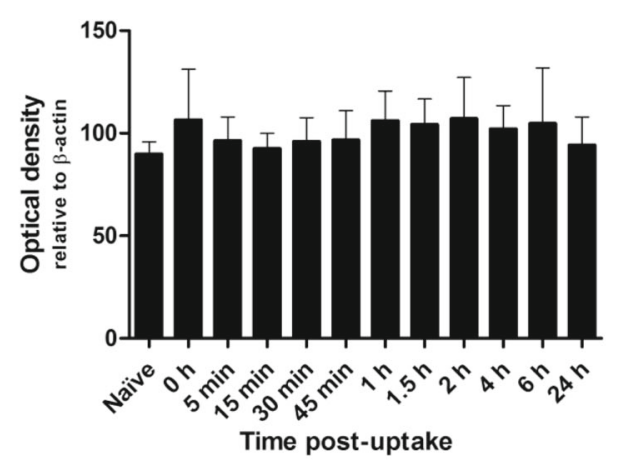

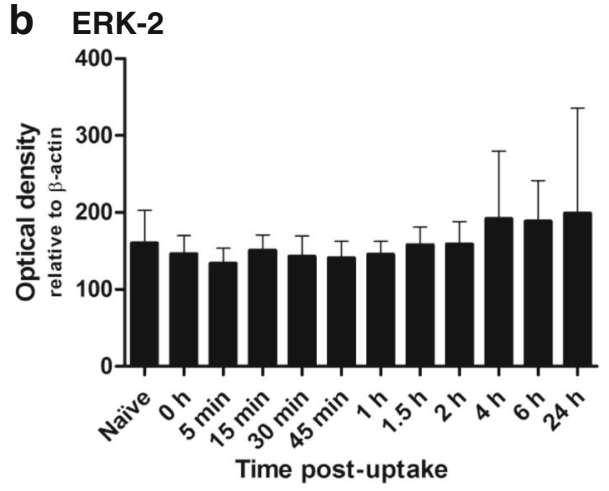

C western blots

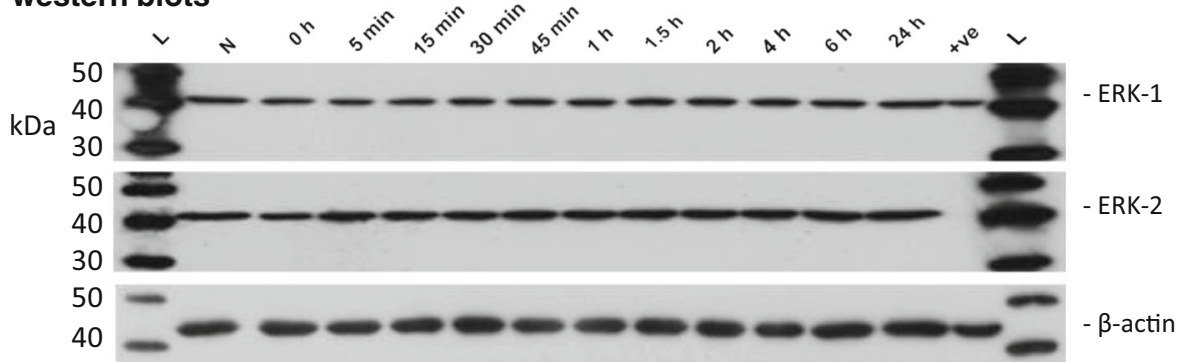

d

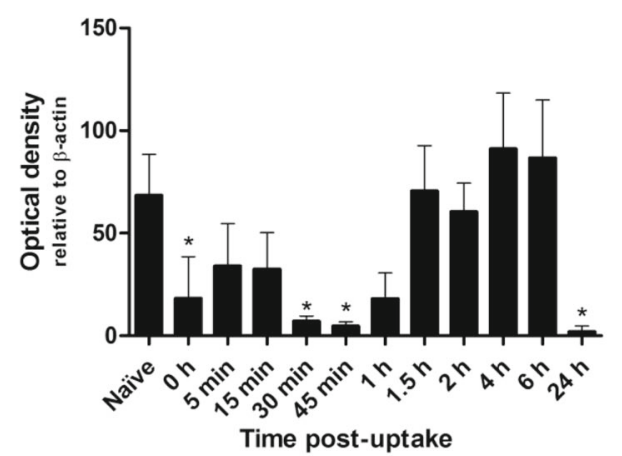

e P-ERK-2

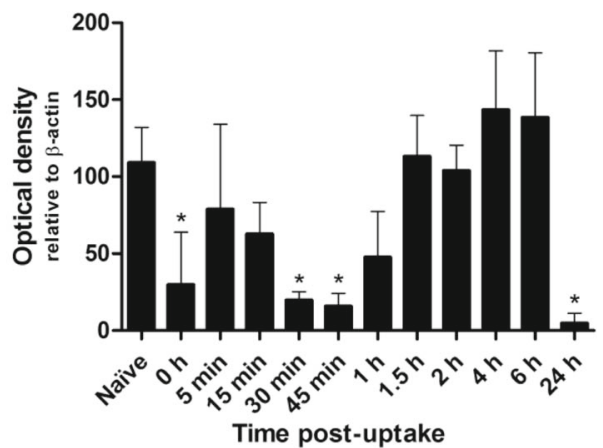

f western blots

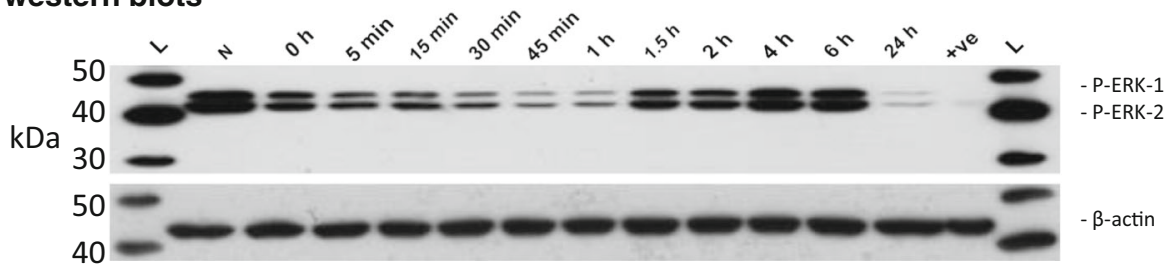

Fig. 3 The expression and levels of activated ERK-1/2 in MH-S cells throughout the course of a $F$. tularensis LVS infection. The expression of ERK-1 (a) and ERK-2 (b) and the levels of activated ERK-1 (c) and ERK-2 (d) were determined at several time points after the 2-h uptake period. Representative western blots are shown below the corresponding graphs. The experiment was performed in triplicate $(n=3)$ and each replicate was performed in duplicate. Error bars show the $95 \%$ confidence interval. Significant difference from naïve levels was determined by one-way ANOVA and a Dunnett's multiple comparison post-test $(*=p<0.05)$. Western blot image: $L-$ MagicMark $^{\mathrm{TM}}$ ladder; $N$ — naïve; $+v e$ - positive control infection of mice. Treatment with the inhibitor resulted in significant decreases in bacterial burden in the spleen and liver but not at primary foci of infection of the lung. The reduction in bacterial burden correlated with a significant decrease in the pro-inflammatory cytokines IFN- $\gamma$, MCP-1, IL-6, and
TNF- $\alpha$, although survival of infected mice was not improved through administration of the inhibitor.

It has previously been reported that the activation of ERK signalling occurs in response to $F$. tularensis infection in vitro and in vivo [9, 19, 31]. Additionally, in vitro studies have 


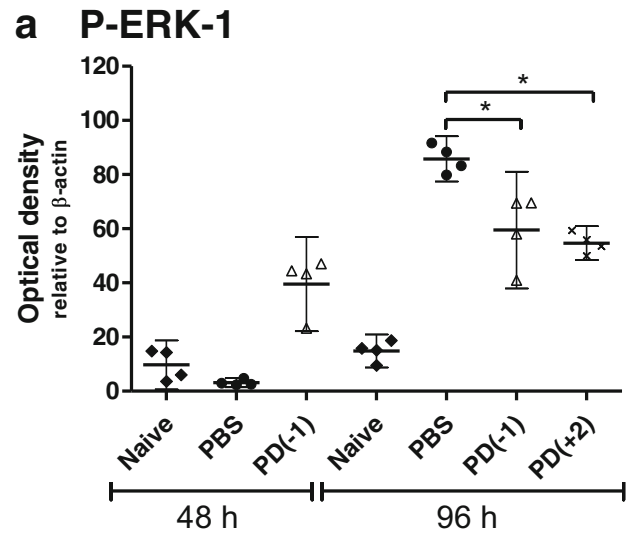

Fig. 4 The effect of PD0325901 treatment $(0.05 \mu \mathrm{M})$ on the activation of ERK-1 and ERK-2 in the lungs of $F$. tularensis LVS infected Balb/c mice. Groups of four mice infected via the intranasal route with $2.5 \times 10^{4}$ CFU F. tularensis LVS were treated daily with PBS from $24 \mathrm{~h}$ pre-challenge (PBS), $\mathrm{PD} 0325901$ from $24 \mathrm{~h}$ pre-challenge $[\mathrm{PD}(-1)]$ or b P-ERK-2

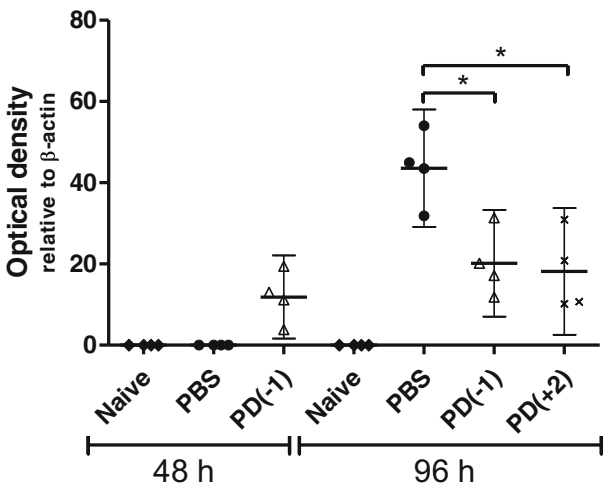

from $48 \mathrm{~h}$ post-challenge $[\mathrm{PD}(+2)]$. Naive mice were used as a control. Detection of phosphorylated ERK-1 and ERK-2 in the lung homogenates of Balb/c mice was carried out by western blotting. Significant difference from PBS-treated group was determined using a one-tailed, unpaired $t$ test $(*=p<0.05)$. Error bars show $95 \%$ confidence intervals
Fig. 5 The effect of PD0325901 treatment $(0.05 \mu \mathrm{M})$ on bacterial burdens and cytokine secretion in the organs of $F$. tularensis LVSinfected Balb/c mice. Groups of four mice infected via the intranasal route with $2.5 \times 10^{4}$ CFU F. tularensis LVS were treated daily with PBS from $24 \mathrm{~h}$ pre-challenge (PBS), PD0325901 from $24 \mathrm{~h}$ prechallenge $[\mathrm{PD}(-1)]$ or from $48 \mathrm{~h}$ post-challenge $[\mathrm{PD}(+2)]$. Naive mice were used as a control. On day 4 post-challenge the organs were removed, homogenised and bacterial burdens were determined (a). The homogenates were also screened for the levels of IL-6 (b), MCP-1 (c), IFN- $\gamma$ (d), and TNF- $\alpha$ (e). Significant difference from PBS-treated group was determined using a one-tailed, unpaired $t$-test $(*=p<0.05)$. Error bars show $95 \%$ confidence intervals
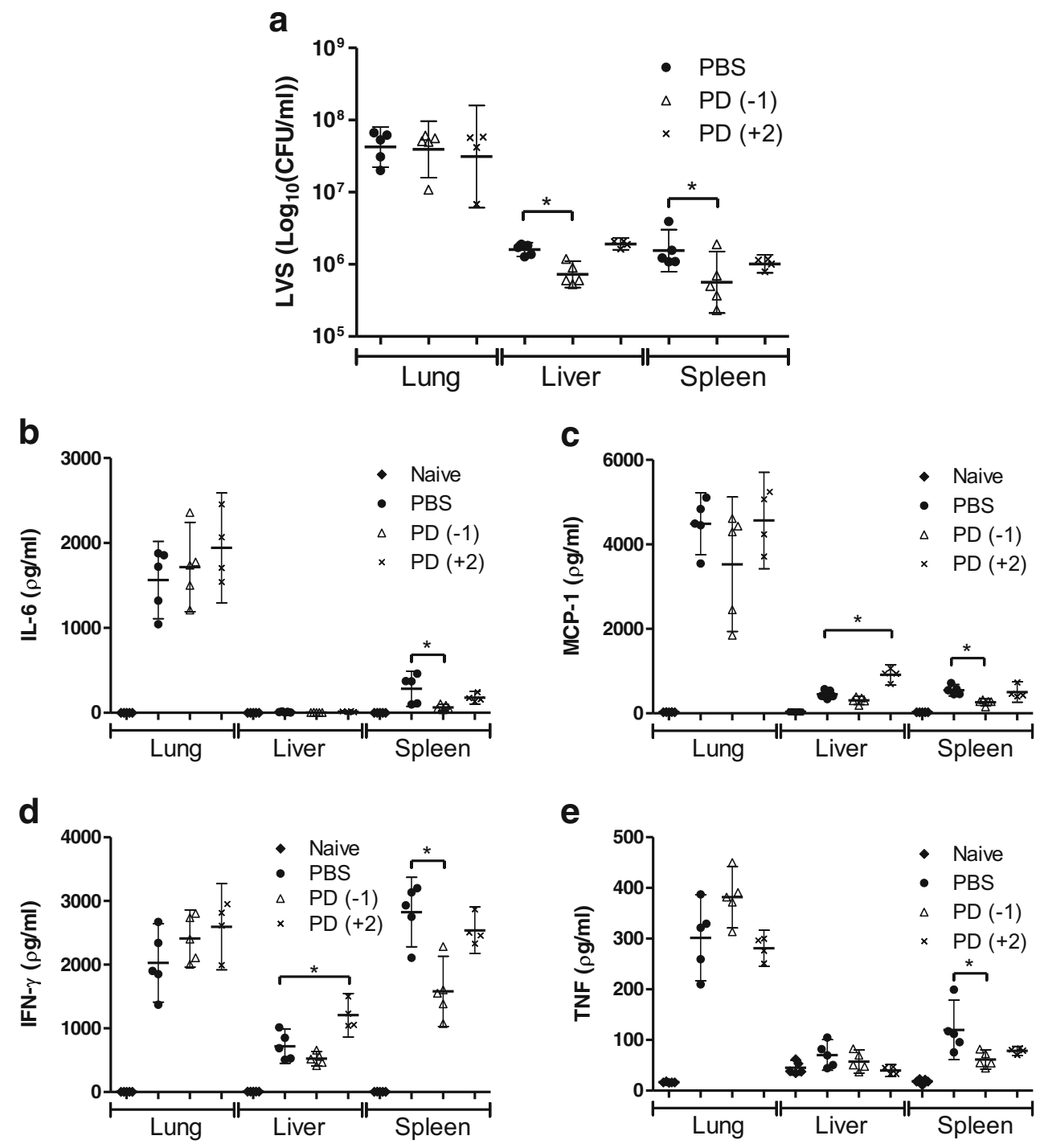


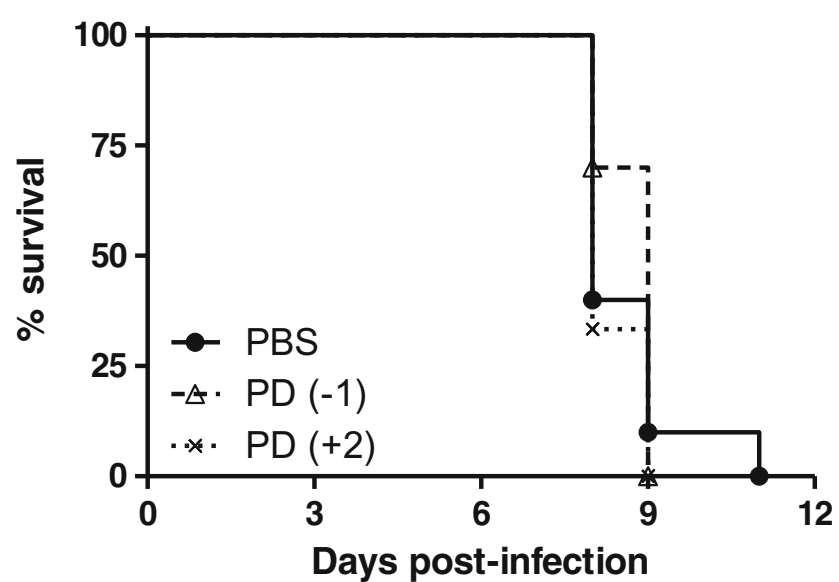

Fig. 6 The effect of PD0325901 treatment $(0.05 \mu \mathrm{M})$ on survival of Balb/c mice infected with $F$. tularensis LVS. Groups of four mice infected via the intranasal route with $2.5 \times 10^{4} \mathrm{CFU} F$. tularensis LVS were treated daily with PBS from $24 \mathrm{~h}$ pre-challenge (PBS), PD0325901 from $24 \mathrm{~h}$ pre-challenge $[\mathrm{PD}(-1)]$ or from $48 \mathrm{~h}$ postchallenge $[\mathrm{PD}(+2)]$. Naive mice were used as a control. The animals were scored daily and were culled upon reaching the humane endpoint

demonstrated that MAPK activation, including ERK activation, plays an important role in F. tularensis LVS infectioninduced apoptosis [32,33]. To our knowledge, however, this is the first assessment of manipulation of ERK as a possible strategy for controlling $F$. tularensis infection. We found that ERK signalling in the lungs of infected mice could be successfully dampened by PD0325901 administration via oral gavage. This did not result in a reduction in bacterial numbers in the lungs (the site of bacterial infection), but there were significantly reduced bacterial burdens in both the liver and spleen of pre-treated mice. This may indicate that the proliferation of the bacteria from the primary site of infection has been reduced.

Sustained ERK activity promotes either intrinsic or extrinsic apoptotic pathways by induction of mitochondrial Cytochrome $\mathrm{C}$ release or caspase- 8 activation, permanent cell cycle arrest, or autophagic vacuolisation [34]. Indeed, it has been suggested that the rapid proliferation of Francisella coincides with the initial activation of the apoptotic signalling pathway [35]. Therefore the relationship between ERK activation and the host mechanism of apoptosis could explain the observed differences in bacterial burden and cytokine response $[33,36]$. In untreated $F$. tularensis infection (and in this study, in PBS-treated mice), the replication of bacteria within the lungs may induce apoptosis of infected cells such as alveolar macrophages via ERK activation, releasing the bacteria for proliferation throughout the host. In contrast, by limiting the activation of ERK through the administration of the inhibitor PD0325901, it is conceivable that the degree of apoptosis could be reduced, preventing cellular release and dissemination of the bacteria. In this context, the lungs act as a trap, with the result that bacterial burdens of this organ are unaffected whereas secondary sites, such as the liver and spleen, demonstrate reduced bacterial burdens as observed here. Further research involving the in vivo administration for the inhibitor and analysis of the presence/absence of apoptotic markers on cellular surfaces is required in order to fully evaluate this hypothesis, which was beyond the scope of this study.

In this study, ERK inhibition did not only alter bacterial burden but also had a significant effect on the proinflammatory secretion profile; specifically it was found that IFN- $\gamma$, MCP-1, IL-6, and TNF- $\alpha$ were all reduced in vivo following pre-treatment with PD0325901. It is well documented that an inflammatory response is essential for pathogen clearance, although it is also clear that pro-inflammatory responses contribute to the tissue damage and pathology seen during infection [6]. Indeed, the overactive immune response seen late during Francisella infection has been hypothesised to be one of the main contributors of detrimental outcome [7,37]. Therefore, in this context, the modulation of the immune response achieved through inhibition of ERK with PD0325901 could be beneficial to survival of the host following infection. However, in this study we demonstrated that although dampening of ERK signalling was observed, along with an associated reduction in cytokine secretion and a decreased systemic proliferation of $F$. tularensis in treated mice, ultimately there was no measurable improvement in survival when compared to PBS-treated mice. This indicates that ERK inhibition alone is not sufficient to improve the outcome of infection with this pathogen. However, it is possible that a combination therapy, for example administering an ERK inhibitor with an antibiotic, could demonstrate a more beneficial outcome of infection. The ERK inhibitor could be used in this context to indirectly reduce systemic spread, whilst a bactericidal antibiotic directly kills the bacteria that remain within the lung. An analogous therapeutic strategy has already been shown to be beneficial in our laboratory for the treatment of the fully virulent SCHUS4 strain of F. tularensis, whereby inhibition of HMGB1, combined with levofloxacin use, significantly increased survival of infected mice compared to controls [7]. In addition, related approaches involving the administration of an antibiotic and an immunodulating therapy have been reported for other bacterial pathogens as well as in the treatment of sepsis [38]. It is important to note that the data generated in this study is derived from a murine model of tularemia as the availability of clinical samples from human cases of the disease is very limited, and it is not possible to conduct clinical trials in humans. However, it is well documented that there are similarities between human tularemia and disease presentation within mouse models, which are well accepted for research on $F$. tularensis [39, 40]. Therefore it is reasonable to extrapolate that targeting 
highly conserved MAPKs in humans would likely have a similar therapeutic benefit.

In conclusion, we have shown that PD0325901 can successfully alter ERK activation during an in vivo $F$. tularensis infection. It caused a reduction in bacterial burden in the liver and spleen if given prior to infection, and also significantly altered the host cytokine profile. The observed difference between mice given PD0325901 prior to or after infection is probably due to the time the compound requires to take effect in vivo and/or as a consequence of the bioavailability of the compound. Ultimately, therapeutic manipulation of ERK did not result in increased survival; however, it may be plausible that PD0325901 and/or other ERK inhibitors would be suitable for assessment within models of infection in combination with an anti-microbial therapy.

Acknowledgments The authors would like to thank the animal technicians that assisted with these studies, and the UK Ministry of Defence for funding this work.

\section{Compliance with ethical standards}

Funding This work was funded by the UK Ministry of Defence.

Conflict of Interest To the authors knowledge there is no conflict of interest arising from the publication of this manuscript.

Ethical approval All procedures performed in studies involving animals were in accordance with the ethical standards of the institution or practice at which the studies were conducted.

Informed consent Not applicable.

Open Access This article is distributed under the terms of the Creative Commons Attribution 4.0 International License (http:// creativecommons.org/licenses/by/4.0/), which permits unrestricted use, distribution, and reproduction in any medium, provided you give appropriate credit to the original author(s) and the source, provide a link to the Creative Commons license, and indicate if changes were made.

\section{References}

1. Alexopoulou L, Holt AC, Medzhitov R, Flavell RA (2001) Recognition of double-stranded RNA and activation of NF-kappa B by Toll-like receptor 3. Nature 413:732-738

2. Jin MS, Kim SE, Heo JY, Lee ME, Kim HM et al (2007) Crystal structure of the TLR1-TLR2 heterodimer induced by binding of a tri-acylated lipopeptide. Cell 130:1071-1082

3. Hirschfeld M, Weis JJ, Toshchakov V, Salkowski CA, Cody MJ et al (2001) Signaling by Toll-like receptor 2 and 4 agonists results in differential gene expression in murine macrophages. Infect Immun 69:1477-1482

4. Droemann D, Goldmann T, Branscheid D, Clark R, Dalhoff K et al (2003) Toll-like receptor 2 is expressed by alveolar epithelial cells type II and macrophages in the human lung. Histochem Cell Biol 119:103-108
5. Sandor F, Buc M (2005) Toll-like receptors. II. Distribution and pathways involved in TLR signalling. Folia Biol 51:188-197

6. D'Elia RV, Harrison K, Oyston PC, Lukaszewski RA, Clark GC (2013) Targeting the "cytokine storm" for therapeutic benefit. Clin Vaccine Immunol 20:319-327

7. D'Elia RV, Laws TR, Carter A, Lukaszewski R, Clark GC (2013) Targeting the "Rising DAMP" during a Francisella tularensis infection. Antimicrob Agents Chemother. doi:10.1128/AAC.01885-12

8. Lembo A, Pelletier M, Iyer R, Timko M, Dudda JC et al (2008) Administration of a synthetic TLR4 agonist protects mice from pneumonic tularemia. J Immunol 180:7574-7581

9. Cole LE, Mann BJ, Shirey KA, Richard K, Yang Y et al (2011) Role of TLR signaling in Francisella tularensis-LPS-induced, antibodymediated protection against Francisella tularensis challenge. J Leukoc Biol 90:787-797

10. Malik M, Bakshi CS, Sahay B, Shah A, Lotz SA et al (2006) Tolllike receptor 2 is required for control of pulmonary infection with Francisella tularensis. Infect Immun 74:3657-3662

11. Macela A, Stulik J, Hernychova L, Kroca M, Krocova Z et al (1996) The immune response against Francisella tularensis live vaccine strain in Lps(n) and Lps(d) mice. FEMS Immunol Med Microbiol 13:235-238

12. Chen WX, KuoLee R, Shen H, Busa M, Conlan JW (2004) Tolllike receptor 4 (TLR4) does not confer a resistance advantage on mice against low-dose aerosol infection with virulent type A Francisella tularensis. Microb Pathog 37:185-191

13. Chen WX, KuoLee R, Shen H, Busa M, Conlan JW (2005) Tolllike receptor 4 (TLR4) plays a relatively minor role in murine defense against primary intradermal infection with Francisella tularensis LVS. Immunol Lett 97:151-154

14. Cole LE, Santiago A, Barry E, Kang TJ, Shirey KA et al (2008) Macrophage proinflammatory response to Francisella tularensis live vaccine strain requires coordination of multiple signaling pathways. J Immunol 180:6885-6891

15. Telepnev M, Golovliov I, Sjostedt A (2005) Francisella tularensis LVS initially activates but subsequently down-regulates intracellular signaling and cytokine secretion in mouse monocytic and human peripheral blood mononuclear cells. Microb Pathog 38:239-247

16. Hong KJ, Wickstrum JR, Yeh HW, Parmely MJ (2007) Toll-like receptor 2 controls the gamma interferon response to Francisella tularensis by mouse liver lymphocytes. Infect Immun 75:53385345

17. Cole LE, Elkins KL, Michalek SM, Qureshi N, Eaton LJ et al (2006) Immunologic consequences of Francisella tularensis live vaccine strain infection: role of the innate immune response in infection and immunity. J Immunol 176:6888-6899

18. Cole LE, Shirey KA, Barry E, Santiago A, Rallabhandi P et al (2007) Toll-like receptor 2-mediated signaling requirements for Francisella tularensis live vaccine strain infection of murine macrophages. Infect Immun 75:4127-4137

19. Thakran S, Li HF, Lavine CL, Miller MA, Bina JE et al (2008) Identification of Francisella tularensis lipoproteins that stimulate the toll-like receptor (TLR)2/TLR1 heterodimer. J Biol Chem 283: 3751-3760

20. Ghonime MG, Mitra S, Eldomany RA, Wewers MD, Gavrilin MA (2015) Inflammasome priming is similar for francisella species that differentially induce inflammasome activation. PLoS One 10: e0127278

21. Elkins KL, Rhinehart-Jones TR, Stibitz S, Conover JS, Klinman DM (1999) Bacterial DNA containing CpG motifs stimulates lymphocyte-dependent protection of mice against lethal infection with intracellular bacteria. J Immunol 162:2291-2298

22. Pyles RB, Jezek GE, Eaves-Pyles TD (2010) Toll-like receptor 3 agonist protection against experimental Francisella tularensis respiratory tract infection. Infect Immun 78:1700-1710 
23. Parsa KVL, Butchar JP, Rajaram MVS, Cremer TJ, Tridandapani S (2008) The tyrosine kinase Syk promotes phagocytosis of Francisella through the activation of Erk. Mol Immunol 45:30123021

24. Rabadi SM, Sanchez BC, Varanat M, Ma Z, Catlett SV et al (2016) Antioxidant defenses of Francisella tularensis modulate macrophage function and production of proinflammatory cytokines. $\mathrm{J}$ Biol Chem 291:5009-5021

25. Zhang P, Katz J, Michalek SM (2009) Glycogen synthase kinase-3 beta (GSK3 beta) inhibition suppresses the inflammatory response to Francisella infection and protects against tularemia in mice. Mol Immunol 46:677-687

26. Roux PP, Blenis J (2004) ERK and p38 MAPK-activated protein kinases: a family of protein kinases with diverse biological functions. Microbiol Mol Biol Rev 68:320-344

27. Zhang W, Liu HT (2002) MAPK signal pathways in the regulation of cell proliferation in mammalian cells. Cell Res 12:9-18

28. Barrett SD, Bridges AJ, Dudley DT, Saltiel AR, Fergus JH et al (2008) The discovery of the benzhydroxamate MEK inhibitors CI1040 and PD 0325901. Bioorg Med Chem Lett 18:6501-6504

29. Thompson N, Lyons J (2005) Recent progress in targeting the Raf/ MEK/ERK pathway with inhibitors in cancer drug discovery. Curr Opin Pharmacol 5:350-356

30. Klimpel GR, Eaves-Pyles T, Moen ST, Taormina J, Peterson JW et al (2008) Levofloxacin rescues mice from lethal intra-nasal infections with virulent Francisella tularensis and induces immunity and production of protective antibody. Vaccine 26:6874-6882

31. Abplanalp AL, Morris IR, Parida BK, Teale JM, Berton MT (2009) TLR-dependent control of Francisella tularensis infection and host inflammatory responses. PLoS ONE 4:e7920
32. Hrstka R, Stulik J, Vojtesek B (2005) The role of MAPK signal pathways during Francisella tularensis LVS infection-induced apoptosis in murine macrophages. Microbes Infect 7:619-625

33. Du K, Zheng Q, Zhou M, Zhu L, Ai B et al (2011) Chlamydial antiapoptotic activity involves activation of the Raf/MEK/ERK survival pathway. Curr Microbiol 63:341-346

34. Cagnol S, Chambard JC (2010) ERK and cell death: mechanisms of ERK-induced cell death - apoptosis, autophagy and senescence. FEBS J 277:2-21

35. Lai XH, Sjostedt A (2003) Delineation of the molecular mechanisms of Francisella tularensis-induced apoptosis in murine macrophages. Infect Immun 71:4642-4646

36. Asim M, Chaturvedi R, Hoge S, Lewis ND, Singh K et al (2010) Helicobacter pylori induces ERK-dependent formation of a Phospho-c-Fos.c-Jun activator protein-1 complex that causes apoptosis in macrophages. J Biol Chem 285:20343-20357

37. Cowley SC (2009) Proinflammatory cytokines in pneumonic tularemia: too much too late? J Leukoc Biol 86:469-470

38. Leelahavanichkul A, Bocharov AV, Kurlander R, Baranova IN, Vishnyakova TG et al (2012) Class B scavenger receptor types I and II and CD36 targeting improves sepsis survival and acute outcomes in mice. J Immunol 188:2749-2758

39. Fritz DL, England MJ, Miller L, Waag DM (2014) Mouse models of aerosol-acquired tularemia caused by Francisella tularensis types A and B. Comp Med 64:341-350

40. Elkins KL, RhinehartJones TR, Culkin SJ, Yee D, Winegar RK (1996) Minimal requirements for murine resistance to infection with Francisella tularesis LVS. Infect Immun 64:3288-3293 